white matter gliosis, 1 of each. Patients with the most favorable outcome had psychomotor type seizures, tumor, and complete resection without complications. Stroke occurred in 2 and infection and hydrocephalus in 1. (Maton B, Jayakar P, Resnick T, Morrison G, Ragheb J, Duchowny M. Surgery for medically intractable temporal lobe epilepsy during early life. Epilepsia Dec 2007;49(1):80-87). (Reprints: Michael Duchowny MD, Miami Children's Hospital, Department of Neurology, 3200 SW $60^{\text {th }}$ Court, Suite 302, Miami, FL 33155).

COMMENT. Cortical resection limited to one temporal lobe for refractory temporal lobe epilepsy is rare in children less than 5 years. This report indicates that surgery in this age group can be associated with favorable outcome, similar to that in older children.

Surgery for epilepsy in children ages 1 - 15 years. A report from Milan, Italy, found that $60 \%$ of 113 patients younger than 16 years (mean age at surgery of 8.8 years) were seizure free following excision of the epileptogenic zone for refractory focal scizures (Cossu $\mathrm{M}$ et al. Epilepsia Dec 2007;49(1):65-72). Variables associated with a significantly lower risk of seizure recurrence were unifocal lesion on MRI, older age at seizure onset, temporal unilobar resection and complete lesionectomy, and glial-neuronal tumor pathology. Results of surgery were strongly dependent on presurgical identification and resection of the epileptogenic zone.

Propeller MRI sequencing for detailed imaging of hippocampal sclerosis. This method is superior to routine MRI sequencies for identifying subtle hippocampal sclerosis (HS), and negates the effects of movement during scans (Eriksson $\mathrm{SH}$ et al. Epilepsia Dec 2007;49(1):33-39). Signs of HS on MRI are increased hippocampal signal on T2-weighted images and loss of hippocampal volume on T1-weighted images. Periodically Rotated Overlapping Parallel Lines with Enhanced Reconstruction (PROPELLER) sequence compensates for head motion during the MRI scan.

\title{
GENETIC VARIATION IN CALCIUM CHANNEL GENE IN IDIOPATHIC GENERALIZED EPILEPSIES
}

Researchers at Women's And Children's Hospital, North Adelaide, and other centers in Australia and Canada screened 240 individuals from 167 families with idiopathic generalized epilepsy and generalized epilepsy with febrile seizures plus (GEFS+) and 95 controls for variants in the CACNAIH gene. They identified 19 novel variants causing amino acid changes associated with the following epilepsy syndromes: childhood absence epilepsy, juvenile absence, juvenile myoclonic, idiopathic generalized with tonic-clonic seizures, and temporal lobe epilepsy. The variants also occurred in unaffected individuals. In some families, the variant segregated with epilepsy, but not in others. It is concluded that variants in $C A C N A I H$ gene that alter channel properties occur in patients with various generalized epilepsy syndromes, contributing to epilepsy susceptibility but not sufficient to cause epilepsy themselves. (Heron SE, Khosravani H, Varela D, et al. Extended spectrum of idiopathic generalized epilepsies associated with CACNA1H functional variants. Ann Neurol Dec 2007;62:560-568). (Respond: Sara E Heron, Department of Genetic Medicine, Women's and Children's Hospital, 72 King William Road, North Adelaide SA 5006, Australia). 
COMMENT. Variants in the CACNAIH gene are associated with a range of generalized epilepsy syndromes, but additional genes or environmental factors also may influence epilepsy susceptibility in some individuals. These genetic variants can contribute to a risk of epilepsy but are not themselves the cause. (Lowenstein D, Messing R. Editorial. Ann Neurol Dec 2007;62:549-551).

\section{ATTENTION DEFICIT DISORDERS}

\section{CONTROLLED STUDY OF GUANFACINE XR IN ADHD}

A multicenter, double-blind, placebo-controlled, fixed-dosage escalation study of an extended release formulation of guanfacine is reported from the Massachusetts General Huspitai, Boston, and other centers in the US and UK. A total of 345 patients aged 6-17 years were randomly assigned to 1 of 3 guanfacine dosage groups $(2,3$, or $4 \mathrm{mg} / \mathrm{each} \mathrm{AM})$ or placebo for 8 weeks. All groups of children taking guanfacine showed significant improvement in hyperactivity/impulsivity and inattentiveness subscales of the ADHD Rating Scale IV, Clinical Global Impression, Parent's Global Assessment, and Conners' Parent and Teacher Rating Scales-Revised. Adverse events included headache, somnolence, fatigue, abdominal pain, and sedation. Treatment was discontinued because of somnolence in $4.2 \%$, sedation in $3.5 \%$, and headache in $1.5 \%$. Somnolence occurred in $15.8-27.6 \%$ patients with doses of $0.04-0.12 \mathrm{mg} / \mathrm{kg}$. Blood pressure and pulse rate decreased as dosages were increased, by a maximum of $-10.1 \mathrm{~mm} \mathrm{Hg}$ (week 4) and $-8 \mathrm{bpm}$ (week 4). Mean changes in ECG (PR and QRS intervals) were unremarkable, mean changes in QTcF intervals were 3.7$9.1 \mathrm{msec}$ (dose related), and no patient had a QT interval $=>480 \mathrm{msec}$. Seven discontinued treatment because of ECG abnormalities, 4 because of QTc interval prolongation, one in each treatment group. Mean changes in height and weight were unremarkable, and group and individual cortisol and human growth hormone levels showed no excessive suppression or elevation. Guanfacine XR was considered safe and effective compared with placebo. (Biederman J, Melmed RD, Patel A, et al. A randomized, double-blind, placebo-controlled study of guanfacine extended release in children and adolescents with attentiondeficit/hyperactivity disorder. Pediatrics January 2008;121:e73-e84). (Respond: Joseph Biedermen MD, Pediatric Psychopharmacology. Yawkey Center, Suitc: 6A. Massachusetts General Hospital, Boston, MA 02114).

COMMENT. Guanfacine is considered a more selective a2-adrenoceptor agonist than clonidine, binding preferentially to receptors in the prefrontal cortex. It has a longer plasma half-life and is less sedating and less hypotensive. The extended release formulation of guanfacine appears to be superior to the immediate release, and is effective in a once daily dosage. In practice, guanfacine XR may be superior to stimulant medications in the younger child with hyperactive behavior and ODD, but the sedative side effect can be troublesome in children of school age. Pretreatment cardiac evaluation with ECG and regular cardiac monitoring are advisable. The potential increase in risk of cardiac complications should limit or discourage the use of a combination of stimulant medication and clonidine or guanfacine. 